\title{
Effect of spin fluctuations on quasiparticle excitations: first-principles theory and application to sodium and lithium
}

\author{
Johannes Lischner,, , 凤 Timur Bazhirov, ${ }^{1}$ Allan H. MacDonald, ${ }^{2}$ Marvin L. Cohen, ${ }^{1}$ and Steven G. Louie ${ }^{1}$ \\ ${ }^{1}$ Department of Physics, University of California, Berkeley, \\ California 94720, USA, and Materials Sciences Division, \\ Lawrence Berkeley National Laboratory, Berkeley 94720, USA. \\ ${ }^{2}$ Department of Physics, The University of Texas at Austin, Austin, Texas, 78712, USA.
}

\begin{abstract}
We present first-principles calculations for quasiparticle excitations in sodium and lithium including the effects of charge and spin fluctuations. We employ the Overhauser-Kukkonen form for the electron self energy arising from spin fluctuations and demonstrate that the coupling of electrons to spin fluctuations gives an important contribution to the quasiparticle lifetime, but does not significantly reduce the occupied bandwidth. Including correlation effects beyond the random-phase approximation in the screening from charge fluctuations yields good agreement with experiment.
\end{abstract}

PACS numbers: 71.20.Dg, 71.10.Ca, 71.15.Qe, 71.45.Gm

Introduction.-The coupling of electrons to spin fluctuations causes many fascinating phenomena: for example, it has been proposed that spin fluctuations can "glue" electrons together to form Cooper pairs giving rise to unconventional high-temperature superconductivity 1 - 4$]$. In particular, spin fluctuations were invoked to explain superconductivity in the cuprates [1-3] and recently also in the iron pnictide and chalcogenide materials [5 [7]. In addition, it is well known that the coupling of spin fluctuations to electrons can affect the electronic effective mass and consequently transport properties and the specific heat.

Theoretically, the effect of spin fluctuations on quasiparticle excitations is usually calculated using model Hamiltonians. Early studies [8-10] constructed empirical theories including spin fluctuations based on the homogeneous electron gas and simple tight-binding models. More recently, many empirical theories involving spin fluctuations were constructed to investigate superconductivity in the cuprates and pnictides. In these theories the spin susceptibility is either parametrized using experimental neutron scattering and nuclear magnetic resonance data [1, 3, 11] or estimated by combining density-functional theory (DFT) with interaction parameters (such as the Hubbard $U$ ) adjusted to reproduce experimental findings $[\underline{6}$, 7].

While the aforementioned theories have been very instructive, their applications have been limited by the availability of concrete experimental data needed to determine their input parameters, supporting the need for a fully first-principles theory without empirical parameters. There have been several attempts to compute the spin fluctuation-electron coupling from first principles. Notably, Winter and coworkers [12, 13] calculated the spin susceptibility and the spin fluctuation-electron self energy from DFT and evaluated the correction to the specific heat for palladium and vanadium. Later studies 14 16] employed a first-principles T-matrix approach to calculate satellites in the photoemission spectrum of nickel and quasiparticle lifetimes in metals. However, such theory requires the solution of a computationally expensive four-point equation and also a correction to account for the double counting of certain Feynman diagrams.

The alkali metals are arguably among the simplest systems in condensed matter theory. Careful comparisons between theory and experiment in these systems has guided progress in understanding electron correlation physics in the itinerant-electron limit. In particular, the Fermi surfaces of sodium and lithium are highly spherical indicating that a description based on the homogeneous electron gas might be valid. Therefore it was surprising when angle-resolved photoemission experiments [17, 18] reported a substantially smaller occupied bandwidth than was found in Hartree calculations on the homogeneous electron gas and also in DFT calculations. In addition, self-energy corrections employing the standard GW approximation to the electron self energy [19, 20], where the self energy is expressed as the product of the interacting Green's function $G$ and the screened Coulomb interaction $W$, could not account for the full reduction of the occupied bandwidth indicating that electron correlation effects not included in these calculations play an essential role in these materials.

Northrup, Hybertsen, and Louie [21, 22] included vertex corrections in the dielectric matrix approximately by computing the charge susceptibility from DFT instead of employing the random-phase approximation (RPA) and found the resulting GW values in good agreement with experiment for the occupied bandwidths of lithium and sodium. At the same time, Zhu and Overhauser 23] found that spin fluctuations within a paramagnon pole model could also explain the reduction of the bandwidth. This difference in the mechanism responsible for the band width reduction in the alkali metals has not yet been resolved. In particular, no first-principles calculation of the self-energy correction arising from spin fluctuations has been reported for the alkali metals.

In this paper, we describe our first-principles calcula- 
tions of the contribution to the self energy arising from spin fluctuations. In particular, we employ the spinfluctuation self energy formalism proposed by Kukkonen and Overhauser 24] which is simpler than the Tmatrix approach since it requires neither the solution of a four-point equation nor a double counting correction. We apply the theory to sodium and lithium, and find that the contribution of spin fluctuations to the reduction of the occupied bandwidth is small and, by itself, cannot explain experimental findings. We also carry out standard GW calculations and GW calculations with a vertex-corrected charge susceptibility, with the latter resulting in a larger reduction of the occupied bandwidth in agreement with experiment and results in Refs. [21, 22].

Methods.-The properties of quasiparticles, such as their energy or lifetime, can be measured in photoemission or tunneling experiments. Mathematically, quasiparticle energies are given by the positions of the poles of the single-particle Green's function and can be determined by solving the Dyson equation

$$
\begin{aligned}
& \left(-\frac{\nabla^{2}}{2}+V_{\mathrm{ion}}(\boldsymbol{r})+V_{H}(\boldsymbol{r})\right) \psi_{n}(\boldsymbol{r})+ \\
& \int d \boldsymbol{r}^{\prime} \Sigma\left(\boldsymbol{r}, \boldsymbol{r}^{\prime}, E_{n}\right) \psi_{n}\left(\boldsymbol{r}^{\prime}\right)=E_{n} \psi_{n}(\boldsymbol{r}),
\end{aligned}
$$

where $V_{\text {ion }}$ and $V_{H}$ denote the external potential due the ionic cores and the Hartree potential, respectively, $E_{n}$ and $\psi_{n}$ are the quasiparticle energy and wave function, and $\Sigma$ denotes the electron self energy.

We approximate the self energy using [23, 25]

$\Sigma\left(\boldsymbol{r}, \boldsymbol{r}^{\prime}, \omega\right)=i \int \frac{d \omega^{\prime}}{2 \pi} e^{-i \delta \omega^{\prime}} G\left(\boldsymbol{r}, \boldsymbol{r}^{\prime}, \omega-\omega^{\prime}\right) V_{\mathrm{eff}}\left(\boldsymbol{r}, \boldsymbol{r}^{\prime}, \omega^{\prime}\right)$,

where $G$ and $V_{\text {eff }}$ denote the single-particle Green's function and the effective interaction between electrons, respectively, and $\delta=0^{+}$. We separate $V_{\text {eff }}$ and subsequently $\Sigma$ into three contributions: a bare Coulomb term $v\left(\boldsymbol{r}, \boldsymbol{r}^{\prime}\right)=1 /\left|\boldsymbol{r}-\boldsymbol{r}^{\prime}\right|$ (resulting in the bare exchange contribution to the self energy), a charge-fluctuation mediated interaction $\delta W_{C}$ (resulting in a correlation contribution to $\Sigma$ arising from charge fluctuations), and a spin-fluctuation mediated interaction $\delta W_{S}$ (resulting in a spin-fluctuation contribution to $\Sigma$ ).

In a standard GW calculation [19, 20], $\delta W_{S}=0$ and $\delta W_{C}=v \chi_{C}^{\mathrm{RPA}} v$ (suppressing all arguments and integrals for clarity) are assumed, where $\chi_{C}^{\mathrm{RPA}}$ denotes the interacting charge susceptibility in the RPA. We will refer to this approximation as $\mathrm{GW}_{\mathrm{RPA}}$.

Following Refs. [21, 22] we include vertex corrections to the dielectric screening by calculating $\chi_{C}$ from DFT, i.e. by solving $\chi_{C}=\chi_{0}+\chi_{0}\left(v+f_{x c}\right) \chi_{C}$, where $\chi_{0}$ denotes the non-interacting Kohn-Sham susceptibility and $f_{x c}\left(\boldsymbol{r}, \boldsymbol{r}^{\prime}\right)=\delta^{2} E_{x c} /\left(\delta \rho(\boldsymbol{r}) \delta \rho\left(\boldsymbol{r}^{\prime}\right)\right)$ with $E_{x c}$ and $\rho(\boldsymbol{r})$ being the exchange-correlation energy within DFT and the electron density, respectively. Note that $\chi_{C}$ is exact if we know the exact $E_{x c}$. The simplest approximation to the exchange-correlation energy is the local density approximation (LDA) 26] and we will refer to this approximation as $\mathrm{GW}_{\mathrm{LDA}}$. Inclusion of vertex corrections in $\chi_{C}$ leads to a better satisfaction of the Ward identity associated with particle conservation [27].

The spin-fluctuation mediated interaction can be approximated [23, 24] using

$$
\delta W_{S}\left(\boldsymbol{r}, \boldsymbol{r}^{\prime}, \omega\right)=3 \int d \boldsymbol{r}_{1} d \boldsymbol{r}_{2} I_{x c}\left(\boldsymbol{r}, \boldsymbol{r}_{1}\right) \chi_{S}\left(\boldsymbol{r}_{1}, \boldsymbol{r}_{2}, \omega\right) I_{x c}\left(\boldsymbol{r}_{2}, \boldsymbol{r}^{\prime}\right),
$$

where $\chi_{S}=\chi_{0}+\chi_{0} I_{x c} \chi_{S}$ denotes the interacting spin susceptibility and $I_{x c}\left(\boldsymbol{r}, \boldsymbol{r}^{\prime}\right)=\delta^{2} E_{x c} /\left(\delta m(\boldsymbol{r}) \delta m\left(\boldsymbol{r}^{\prime}\right)\right)$ with $m(\boldsymbol{r})$ denoting the spin density. The factor of 3 results from the vector boson nature of the spin fluctuations.

This intuitively appealing expression for the self energy arising from spin fluctuations was first derived by considering the effective interaction between electrons in a homogeneous electron gas including exchange and correlation effects [24]. Later 25] it was found that the same expression may be obtained from an analysis of Feynman diagrams [42].

Computational details. - We carry out DFT-LDA calculations using a plane-wave basis and normconserving pseudopotentials as implemented in the Quantum Espresso program package [28]. Our plane wave cutoff is 30 Ry. For sodium we choose the unit cell corresponding to $r_{s}=3.93$ and for lithium corresponding to $r_{s}=3.26\left[r_{s}\right.$ is related to the valence charge density $n$ via $n=3 /\left(4 \pi\left(r_{s} a_{B}\right)_{s}^{3}\right)$ with $a_{B}$ being the Bohr radius]. The self energy is calculated using the BerkeleyGW [29] program package. For the calculation of the susceptibilities and the self energies we use $16 \times 16 \times 16 \mathrm{k}$-point sampling of the Brillouin zone. In our first-principles calculations, we do not employ a generalized plasmon-pole model for the interacting charge and spin susceptibilities, but sample these quantities along the real frequency axis. We use fine sampling with a step size of $0.1 \mathrm{eV}$ up to a lower cutoff of $30 \mathrm{eV}$ and then coarser sampling up to $60 \mathrm{eV}$. A broadening of $0.15 \mathrm{eV}$ is used as well as 30 empty states in the calculation of the dielectric matrix and the self energy. In this work, we employ a one-shot procedure to calculate the self energy. The effect of self-consistency on the occupied band width of simple metals was investigated in Ref. 21] and found to be quite small.

Sodium.-The occupied bandwidth of sodium in DFT is $3.19 \mathrm{eV}$. Figure 1(a) shows the self-energy correction (evaluated "on-shell", i.e. at the mean-field energy) to the DFT-LDA band structure from charge fluctuations (including the bare exchange) and from spin fluctuations. We find a reduction of the occupied bandwidth with charge fluctuations giving a significantly larger contribution than spin fluctuations. In agreement with previous 

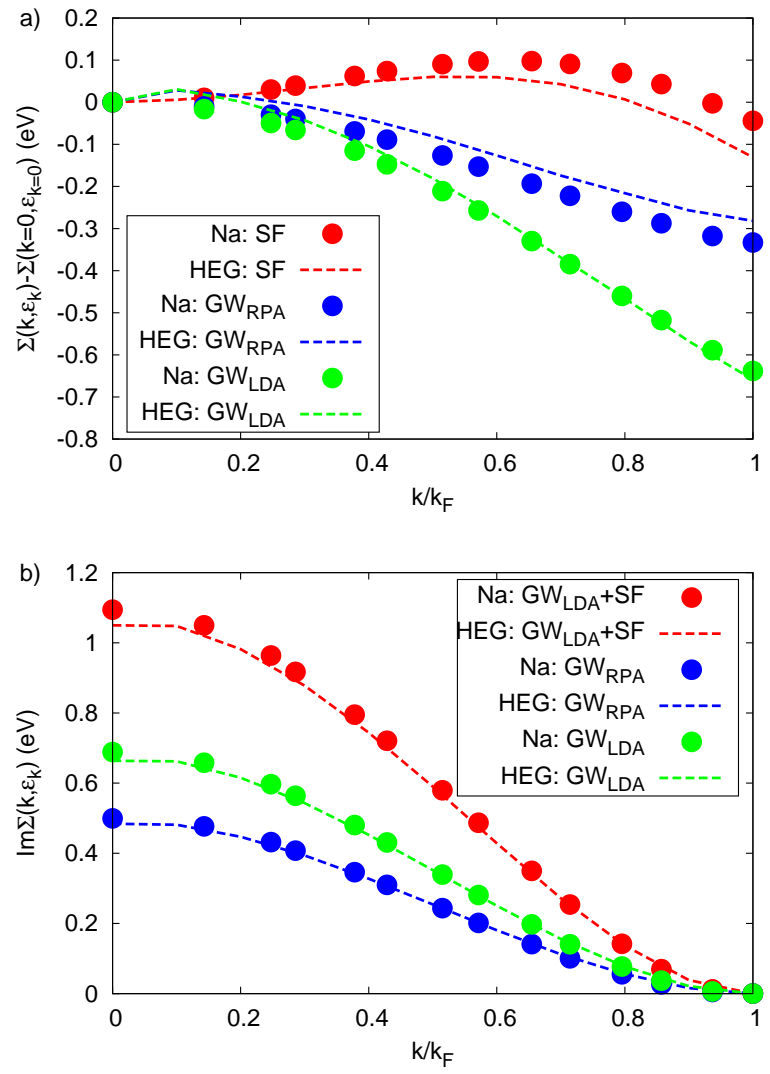

FIG. 1: a) On-shell self-energy corrections for the occupied states in sodium. The dashed curves are results for the homogeneous electron gas (HEG) with $r_{s}=3.93$, while the circles are results from $a b$ initio calculations. The $\mathrm{GW}_{\mathrm{RPA}}$ theory (blue), GW $\mathrm{LDA}_{\text {LD }}$ theory (green), and the spin-fluctuation self energy (red) are shown. b) Imaginary part of the on-shell self energy for the occupied states in sodium. The labels are the same as in a).

calculations 21, 22], we find that vertex corrections in the charge susceptibility are very important (increasing the bandwidth reduction by a factor of two compared to the standard $\mathrm{GW}_{\mathrm{RPA}}$ result): standard $\mathrm{GW}_{\mathrm{RPA}}$ theory gives a reduction of $0.31 \mathrm{eV}$, while $\mathrm{GW}_{\mathrm{LDA}}$ yields a reduction of $0.63 \mathrm{eV}$ resulting in an occupied bandwidth in good agreement with the experimental findings [17, 18]. See Table [1. The contribution from spin fluctuations to the bandwidth reduction is very small, less than $0.1 \mathrm{eV}$.

To understand these results we observe that retaining only the bare exchange contribution to the self energy results in a drastic increase of the occupied bandwidth by $3.30 \mathrm{eV}$ as expected from usual Hartree-Fock theory. Inclusion of screening by charge fluctuations has the opposite effect yielding a net reduction of the bandwidth. The RPA underestimates the screening which explains the larger bandwidth reduction in $\mathrm{GW}_{\mathrm{LDA}}$ theory [22].

Figure 1(a) also shows the result from a self-energy calculation for the homogeneous electron gas (jellium) with
TABLE I: Occupied bandwidth of sodium obtained from onshell and off-shell evaluations of the self energy. We also give results with approximate self-consistency (sc) achieved by shifting the mean-field energies. All energies are given in $\mathrm{eV}$.

\begin{tabular}{c|ccc}
\hline \hline & on-shell & off-shell & off-shell + sc \\
\hline GW $_{\text {RPA }}$ & 2.86 & 3.00 & 2.98 \\
GW $_{\text {LDA }}$ & 2.55 & 2.83 & 2.80 \\
GW & 2.51 & 2.85 & 2.78 \\
\hline exp. [17] & 2.5 & & \\
exp. [18] & 2.65 & & \\
DFT-LDA & 3.19 & & \\
\hline \hline
\end{tabular}

$r_{s}=3.93$ corresponding to the valence charge density of sodium, $n=3 /\left(4 \pi\left[r_{s} a_{B}\right]^{3}\right)$ with $a_{B}$ being the Bohr radius. The occupied bandwidth in Hartree theory is 3.15 $\mathrm{eV}$. This agrees very well with the $a b$ initio DFT-LDA result indicating that corrections caused by the inhomogeneity of the crystalline potential are very small. Fig. 1 shows good agreement between the on-shell self energies from the $a b$ initio calculation and jellium.

If the exact self energy was known, quasiparticle properties should be calculated "off-shell", i.e. the quasiparticle equation, Eq. (1), should be solved and the self energy should be evaluated at the quasiparticle energy. It has been argued 30, 31], however, that for approximate self energies quasiparticle properties should be determined by evaluating the self energy "on the shell" to avoid mixing different orders of perturbation theory. Table I shows that off-shell calculations result in a larger occupied bandwidth than on-shell calculations. The ratio of the bandwidth reductions in off-shell and on-shell calculations is approximately equal to the renormalization constant $Z_{\boldsymbol{k}}=\left[1-\partial \Sigma_{\boldsymbol{k}}\left(E_{\boldsymbol{k}}^{q p}\right) / \partial \omega\right]^{-1}$, which is about 0.6 for the occupied states of sodium.

According to Eq. (2), the self energy should be calculated using the interacting Green's function. To approximately account for this self-consistency requirement we have shifted all mean-field energies such that the resulting quasiparticle energy agrees with the shifted meanfield energy [32] at the Fermi level. Table \ shows that self-consistency only leads to very small changes in the occupied bandwidth.

We employed the jellium model to investigate the effect of additional approximations to the self energy. In standard first-principles $\mathrm{GW}_{\mathrm{RPA}}$ calculations, one sometimes employs a generalized plasmon-pole model [19] to extend the static inverse dielectric matrix to finite frequencies. In this model the imaginary part of the inverse dielectric function for each $\boldsymbol{G}$ and $\boldsymbol{G}^{\prime}$ component is assumed to be a simple delta-function, i.e. $\operatorname{Im} \epsilon_{G G^{\prime}}^{-1}(\boldsymbol{q}, \omega) \propto$ $\delta\left(\omega-\omega_{G G^{\prime}}(\boldsymbol{q})\right)$ with $\omega_{G G^{\prime}}(\boldsymbol{q})$ denoting the effective plasmon frequency. Fig. 2(a) shows that the plasmonpole model reproduces the self-energy shifts arising from charge fluctuations quite well. Zhu and Overhauser 23] 
employed a similar paramagnon-pole model to simplify the calculation of the spin-fluctuation self energy: they assumed that the imaginary part of the interacting spin susceptibility can be represented by a single mode, the paramagnon. However, in contrast to the plasmon which cannot decay into particle-hole pairs for small wave vectors, the paramagnon has a linear acoustic-like dispersion (as determined by the f-sum rule [23]) and can decay into particle-hole pairs: Fig. 2(b) shows the imaginary part of the spin, charge, and non-interacting susceptibilities at $q / q_{F}=0.6$. The charge susceptibility has a sharp plasmon peak at $\sim 7 \mathrm{eV}$ that lies outside the particle-hole continuum given by the non-interacting susceptibility. The spin susceptibility has significant overlap with the non-interacting susceptibility and exhibits a broad structure. In contrast to the charge susceptibility, the spin susceptibility is not well represented by a single sharp mode.

As a consequence, the spin-fluctuation self energy with a paramagnon-pole model gives very different results from the theory without this approximation, see Fig. 2(a). It results in a drastic narrowing of the occupied bandwidth by $\sim 1.4 \mathrm{eV}$. While Zhu and Overhauser [23] empirically correct for the finite lifetimes of the paramagnon, it is likely that their predicted bandwidth narrowing of $0.7 \mathrm{eV}$ is also spuriously large and caused by the paramagnon-pole approximation.

Figure 2 also shows that for the $\mathrm{GW}_{\mathrm{LDA}}$ theory the plasmon-pole approximation leads to a further reduction of the occupied bandwidth by $\sim 0.2 \mathrm{eV}$. The resulting bandwidth (calculated "off-shell") agrees well with previous first-principles results [21, 22].

Figure 1(b) on the other hand shows that spin fluctuations contribute significantly to the line width $\left[2 \operatorname{Im} \Sigma_{\boldsymbol{k}}\left(\epsilon_{\boldsymbol{k}}\right)\right]$ of quasiparticles. In particular, at the bottom of the band, $k=0$, the quasiparticle line width is a factor of 2 larger than the $\mathrm{GW}_{\mathrm{RPA}}$ value when vertex corrections in $\chi_{C}$ and spin-fluctuations are included. This agrees well with the experimental findings [16, 34, 35].

Lithium. - In contrast to sodium, the occupied bandwidth resulting from Hartree theory applied to the homogeneous electron gas, $4.65 \mathrm{eV}$, is much larger than the value obtained in a DFT-LDA calculation including the crystalline potential, $3.45 \mathrm{eV}$. This shows that - even though the Fermi surface is spherical to a high degree [36] - crystal effects are very important in lithium.

Table I shows our results for the occupied bandwidth of lithium. Again, the occupied bandwidth reduction in $\mathrm{GW}_{\mathrm{RPA}}$ theory is too small to explain the experimental finding. Adding vertex correction in $\chi_{C}$ yields good agreement with experiment [37], while spin fluctuations only lead to a small change in the occupied bandwidth.

Conclusions.-We have calculated the effect of spin and charge fluctuations on quasiparticle excitations in alkali metals from first principles. In contrast to previous calculations [23], we find that spin fluctuations contribute
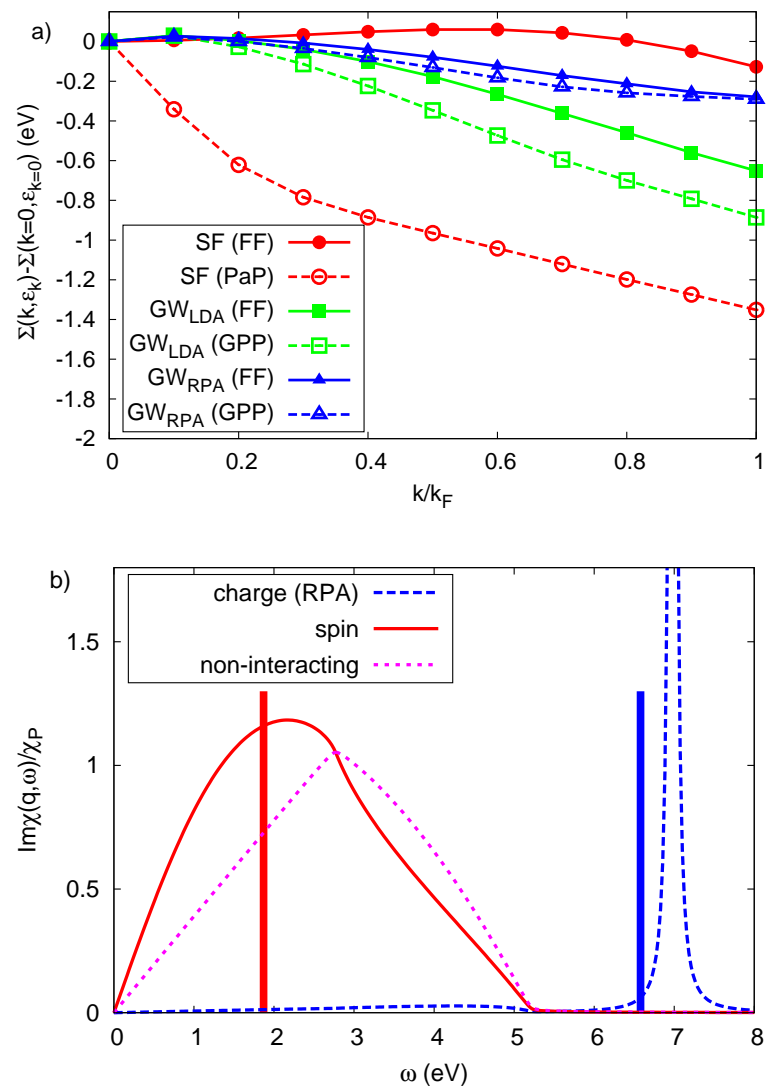

FIG. 2: a) Comparison of the on-shell self-energy corrections for the occupied states of jellium with $r_{s}=3.93$ (corresponding to sodium) from full-frequency and simplified calculations using the generalized plasmon-pole (GPP) and paramagnon-pole $(\mathrm{PaP})$ models for the $\mathrm{GW}_{\mathrm{RPA}}$ self energy (blue), GW LDA self energy (green), and spin-fluctuation self energy (red). b) Frequency-dependent imaginary parts of the charge (blue dashed curve), spin (red solid curve), and noninteracting (magenta dotted curve) susceptibilities for jellium at $q / q_{F}=0.6$. The susceptibilities are divided by the Pauli susceptibility $\chi_{P}=-k_{F} / \pi^{2}$. The vertical lines denote the locations of the delta-function peaks in a plasmon-pole model (blue) [33] and a paramagnon-pole model (red) [23].

TABLE II: Occupied bandwidth of lithium obtained from onshell and off-shell evaluations of the self energy. We also give results with approximate self-consistency (sc) achieved by shifting the mean-field energies. All energies are given in $\mathrm{eV}$.

\begin{tabular}{c|ccc}
\hline \hline & on-shell & off-shell & off-shell + sc \\
\hline GW $_{\text {RPA }}$ & 3.19 & 3.29 & 3.30 \\
GW $_{\text {LDA }}$ & 2.83 & 3.09 & 3.10 \\
GW $_{\text {LDA }}+\mathrm{SF}$ & 2.87 & 3.24 & 3.08 \\
\hline exp. [37] & 2.86 & & \\
DFT-LDA & 3.45 & & \\
\hline \hline
\end{tabular}


little to the observed bandwidth reduction compared to mean-field results. Instead, as observed in Refs. 21, 22] inclusion of vertex corrections in the dielectric screening gives agreement with experimental bandwidths. Previous studies which included vertex corrections in both the dielectric screening and the self energy found that agreement with experiment worsens [38, 39]. Other studies reported cancellations between self-energy vertex corrections and selfconsistency effects [40, 41]. Further work is necessary to clarify this issue. We also find that spin fluctuations give an important contribution to the line width and lifetime of the quasiparticle excitations. We note that the first-principles framework presented here can be applied to materials with d-electrons where spin fluctuations are expected to play an essential role, such as pnictide superconductors and ferromagnetic metals.

S. G. L. acknowledges support by a Simons Foundation Fellowship in Theoretical Physics. This work was supported by NSF Grant No. DMR10-1006184 (numerical simulations of the alkali metals) and by the Director, Office of Science, Office of Basic Energy Sciences, Division of Materials Sciences and Engineering Division, US Department of Energy under Contract No. DE-AC0205CH11231 (software development of electron correlation effects).

* Electronic address: jlischner@civet.berkeley.edu

[1] T. Dahm, V. Hinkov, S. V. Borisenko, A. A. Kordyuk, V. B. Zabolotnyy, J. Fink, B. Buechner, D. J. Scalapino, W. Hanke, and B. Keimer, Nature Physics 5, 217 (2009).

[2] M. L. Tacon, G. Ghiringhelli, J. Chaloupka, M. M. Sala, V. Hinkov, M. W. Haverkort, M. Minola, M. Bakr, K. J. Zhou, S. Blanco-Canosa, et al., Nature Physics 7, 725 (2011).

[3] P. Monthoux, A. V. Balatsky, and D. Pines, Phys. Rev. Lett. 67, 3448 (1991).

[4] D. J. Scalapino, Rev. Mod. Phys. 84, 1383 (2012).

[5] I. I. Mazin, D. J. Singh, M. D. Johannes, and M. H. Du, Phys. Rev. Lett. 101, 057003 (2008).

[6] K. Kuroki, S. Onari, R. Arita, H. Usui, Y. Tanaka, H. Kontani, and H. Aoki, Phys. Rev. Lett. 101, 087004 (2008).

[7] S. Graser, A. F. Kemper, T. A. Maier, H.-P. Cheng, P. J. Hirschfeld, and D. J. Scalapino, Phys. Rev. B 81, 214503 (2010).

[8] S. Doniach and S. Engelsberg, Phys. Rev. Lett. 17, 750 (1966).

[9] N. F. Berk and J. R. Schrieffer, Phys. Rev. Lett. 17, 433 (1966).

[10] J. R. Schrieffer, Phys. Rev. Lett. 19, 644 (1967).

[11] A. Helmes, R. Grein, and M. Eschrig, Phys. Rev. Lett. 106, 047003 (2011).

[12] E. Stenzel, H. Winter, Z. Szotek, and W. M. Temmerman, Z. Phys. B - Condensed Matter 70, 173 (1988).
[13] E. Stenzel and H. Winter, J. Phys. F: Met. Phys. 16, 1789 (1986).

[14] M. Springer, F. Aryasetiawan, and K. Karlsson, Phys. Rev. Lett. 80, 2389 (1998).

[15] V. P. Zhukov, E. V. Chulkov, and P. M. Echenique, Phys. Rev. Lett. 93, 096401 (2004).

[16] V. P. Zhukov, E. V. Chulkov, and P. M. Echenique, Phys. Rev. B 72, 155109 (2005).

[17] E. Jensen and E. W. Plummer, Phys. Rev. Lett. 55, 1912 (1985).

[18] I.-W. Lyo and E. W. Plummer, Phys. Rev. Lett. 60, 1558 (1988).

[19] M. S. Hybertsen and S. G. Louie, Phys. Rev. B 34, 5390 (1986).

[20] L. Hedin and S. Lundqvist, Solid State Physics 23, 1 (1969).

[21] J. E. Northrup, M. S. Hybertsen, and S. G. Louie, Phys. Rev. Lett. 59, 819 (1987).

[22] J. E. Northrup, M. S. Hybertsen, and S. G. Louie, Phys. Rev. B 39, 8198 (1989).

[23] X. Zhu and A. W. Overhauser, Phys. Rev. B 33, 925 (1986).

[24] C. A. Kukkonen and A. W. Overhauser, Phys. Rev. B 20, 550 (1979).

[25] G. Vignale and K. S. Singwi, Phys. Rev. B 32, 2156 (1985).

[26] W. Kohn and L. J. Sham, Phys. Rev. 140, A1133 (1965).

[27] G. Strinati, H. Mattausch, and W. Hanke, Phys. Rev. B 25, 2867 (1982).

[28] P. Giannozzi, S. Baroni, N. Bonini, M. Calandra, R. Car, C. Cavazzoni, D. Ceresoli, G. L. Chiarotti, M. Cococcioni, I. Dabo, et al., Journal of Physics: Condensed Matter 21, 395502 (2009).

[29] J. Deslippe, G. Samsonidze, D. A. Strubbe, M. Jain, M. L. Cohen, and S. G. Louie, Comput. Phys. Commun. 183, 1269 (2012).

[30] T. M. Rice, Ann. Phys. (N.Y.) 31, 100 (1965).

[31] Y. Zhang and S. D. Sarma, Phys. Rev. B 71, 045322 (2005).

[32] L. Hedin, J. Phys.: Condens. Matter 11, 489 (1999).

[33] B. I. Lundqvist, Phys. Kondens. Mater. 6, 193 (1967).

[34] E. W. Plummer, Phys. Scripta T17, 186 (1987).

[35] D. R. Penn, Phys. Rev. B 22, 2677 (1980).

[36] J. Callaway, Phys. Rev. 124, 1824 (1961).

[37] R. M. Watson, Ph.D. Thesis, University of Pennsylvania (1992).

[38] G. D. Mahan and B. E. Sernelius, Phys. Rev. Lett. 62, 2718 (1989).

[39] A. J. Morris, M. Stankovski, K. T. Delaney, P. Rinke, P. Garcia-Gonzalez, and R. W. Godby, Phys. Rev. B 276, 155106 (2007).

[40] B. Holm and U. von Barth, Physica Scripta T109, 135 (2004).

[41] B. Holm and U. von Barth, Phys. Rev. B 57, 2108 (1998).

[42] Refs. 24, 25] suggested further exchange and correlations effects may be included into the charge-fluctuation mediated interaction, $\delta W_{C}=\left(v+f_{x c}\right) \chi_{C}\left(v+f_{x c}\right)$. In agreement with previous studies 23], we find however that use of this interaction worsens the agreement with experiment compared to the $\mathrm{GW}_{\mathrm{LDA}}$ theory. 